Annals of Pure and Applied Mathematics

Vol. 14, No. 1, 2017, 45-52

ISSN: 2279-087X (P), 2279-0888(online)

Published on 3 July 2017

Annals of

www.researchmathsci.org

DOI: http://dx.doi.org/10.22457/apam.v14n1a6

Pure and Applied

Mathematics

\title{
Some New Results on Sum Divisor Cordial Graphs
}

\author{
A.Sugumaran ${ }^{1}$ and K.Rajesh ${ }^{2}$ \\ Department of Mathematics, Government Arts College \\ Tiruvannamalai-606603, Tamilnadu, India. \\ ${ }^{1}$ E-mail:sugumaranaruna@gmail.com \\ Corresponding author. ${ }^{2}$ E-mail:k.rajesh3429@gmail.com
}

Received 14 May 2017; accepted 29 June 2017

Abstract. A sum divisor cordial labeling of a graph $G$ with vertex set $V$ is a bijection $\mathrm{f}$ from $V$ to $\{1,2, \ldots,|V(G)|\}$ such that each edge uv assigned the label 1 if 2 divides $f(u)+f(v)$ and 0 otherwise. Further, the number of edges labeled with 0 and the number of edges labeled with 1 differ by at most 1 . A graph with a sum divisor cordial labeling is called a sum divisor cordial graph. In this paper, we prove that Swastik graph $S w_{n}$, path union of finite copies of Swastik graph $S w_{n}$, cycle of $k$ copies of Swastik graph $S w_{n}$ ( $k$ is odd), Jelly fish $J(n, n)$ and Petersen graph are sum divisor cordial graphs.

Keywords: Divisor cordial labeling, sum divisor cordial labeling.

AMS Mathematics Subject Classification (2010): $05 \mathrm{C} 78$

\section{Introduction}

By a graph, we mean a finite undirected graph without loops or multiple edges. For standard terminology and notations related to graph theory we refer [4]. A labeling of graph is a map that carries the graph elements to the set of numbers, usually to the set of non-negative or positive integers. If the domain is the set of edges, then we speak about edge labeling. If the labels are assigned to both vertices and edges, then the labeling is called total labeling. Cordial labeling is extended to divisor cordial labeling, prime cordial labeling, total cordial labeling, Fibonacci cordial labeling etc. The total cordial labeling concept is further extended to edge magic total labeling, edge trimagic total labeling, 3-equitable and total magic cordial labeling etc.

Girija and Elumalai [3] have discussed edge magic total labeling of some standard graphs. Jayasekaran and Little flower [5] discussed the edge trimagic total labeling of Mobius ladder, book and dragon graphs. 3-Equitable and total magic cordial labeling of some standard graphs are discussed by Avudainagai et al. [1]. 
Some New Results on Sum Divisor Cordial Graphs

Varatharajan et al. [12] introduced the concept of divisor cordial labeling. Vaidya and shah [11] proved that some star and bistars related graphs are divisor cordial labeling. Deshmukh and Shaikh [10] proved that Tadpole and olive tree graphs are mean cordial graphs. Rokad and Godasara [9] have discussed the Fibonacci cordial labeling of some special graphs. For dynamic survey of various graph labeling, we refer to Gallian [2].

Lourdusamy and Patrick [8] introduced the concept of sum divisor cordial labeling. In this paper we investigate the sum divisor cordial labeling behavior of Swastik graph $S w_{n}$, path union of finite copies of Swastik graph $S w_{n}$, cycle of $k$ copies of Swastik graph $S w_{n}$ ( $k$ is odd), Jelly fish $J(n, n)$ and Petersen graph.

Definition 1.1.[12] Let $G=(V(G), E(G))$ be a simple graph and let $f: V(G) \rightarrow\{1,2, \ldots,|V(G)|\}$ be a bijection. For each edge $u v$, assign the label 1 if either $f(u) \mid f(v)$ or $f(v) \mid f(v)$ and the label 0 otherwise. The function $f$ is called a divisor cordial labeling if $\left|e_{f}(0)-e_{f}(1)\right| \leq 1$. A graph which admits a divisor cordial labeling is called a divisor cordial graph.

Definition 1.2. [8] Let $G=(V(G), E(G))$ be a simple graph and let $f: V(G) \rightarrow\{1,2, \ldots,|V(G)|\}$ be a bijection. For each edge $e=u v$, assign the label 1 if either $2 \mid(f(u)+f(v))$ and assign the label 0 otherwise. The function $f$ is called a sum divisor cordial labeling if $\left|e_{f}(0)-e_{f}(1)\right| \leq 1$. A graph which admits a sum divisor cordial labeling is called a sum divisor cordial graph.

Definition 1.3. [6] Consider four copies of cycle $C_{4 n}(n \succ 1)$.

Let $V\left(C_{4 n}\right)=\left\{v_{i, j}: 1 \leq i \leq 4 ; 1 \leq j \leq 4 n\right\}$. We combine the following vertices by a single vertex as $v_{i, 4 n}=v_{i+1,1}$ for $i=1,2,3$ and $v_{4,4 n}=v_{1,1}$. In each cycle we bend the graph 90 degree towards clockwise at the vertices $v_{i, n+1}, v_{i, 2 n+1}, v_{i, 2 n+2}, v_{i, 3 n+1}(i=1,2,3,4)$, then the resulting graph is in the form of Swastik and it is denoted by $S w_{n}$. Obviously, $|V(G)|=16 n-4$ and $|E(G)|=16 n$.

Definition 1.4. [6] Let $G$ be a graph and let $G_{1}=G_{2}=\cdots=G_{n}=G$, where $n \geq 2$. Then the graph obtained by adding an edge from each $G_{i}$ to $G_{i+1}(1 \leq i \leq n-1)$ is called the path union of $G$.

Definition 1.5. [6] For a cycle $C_{n}$, each vertex of $C_{n}$ is replaced by connected graphs $G_{1}, G_{2}, \ldots, G_{n}$ and is known as cycle of graphs. We shall denote it by $C\left(G_{1}, G_{2}, \ldots, G_{n}\right)$. 


\section{A.Sugumaran and K.Rajesh}

If we replace each vertex of $C_{n}$ by a graph $G$, then we denote such cycle of graphs as $C(n \cdot G)$.

Definition 1.6. [7] Let $C_{4}$ be a cycle with vertex set $\left\{v_{1}, v_{2}, v_{3}, v_{4}\right\}$. The Jelly fish graph $J(m, n)$ is obtained by joining $v_{1}$ and $v_{3}$ by an edge, also appending $m$ pendant edges at $v_{2}$ and $n$ pendant edges at $v_{4}$.

\section{Main results}

Theorem 2.1. A Swastik graph $S w_{n}$ is a sum divisor cordial graph.

Proof: Let $v_{k, i}(1 \leq k \leq 4 ; 1 \leq i \leq 4 n)$ be the vertices of $k^{\text {th }}$ copy of $C_{4 n}$ in Swastik graph $S w_{n}$, where $v_{k, 4 n}=v_{k+1,1}(1 \leq k \leq 3)$ and $v_{4,4 n}=v_{1,1}$.

We define the vertex labeling $f: V(G) \rightarrow\{1,2, \ldots,|V(G)|\}$ as follows.

$$
\begin{aligned}
& f\left(v_{1,1}\right)=f\left(v_{4,4 n}\right)=1, \quad f\left(v_{1,4 n}\right)=f\left(v_{2,1}\right)=2, \\
& f\left(v_{3,1}\right)=f\left(v_{2,4 n}\right)=4, \quad f\left(v_{4,1}\right)=f\left(v_{3,4 n}\right)=3, \\
& f\left(v_{1, i}\right)=i+3 ; i=2,3, \ldots, 4 n-1, \\
& f\left(v_{2, i}\right)=4 n+1+i ; i=2,3, \ldots, 4 n-1, \\
& f\left(v_{3, i}\right)=2(4 n+i)-2 ; i=2,3, \ldots, 2 n, \\
& f\left(v_{3, i}\right)=2(2 n+i)-1 ; i=2 n+1, \ldots, 4 n-1, \\
& f\left(v_{4, i}\right)=2(6 n+i)-5 ; i=2,3, \ldots, 2 n, \\
& f\left(v_{4, i}\right)=2(4 n+i)-2 ; i=2 n+1, \ldots, 4 n-1 .
\end{aligned}
$$

From the above labeling pattern, we have $\left|e_{f}(0)-e_{f}(1)\right| \leq 1$.

Hence a Swastik graph $S w_{n}$ is a sum divisor cordial graph.

Example 2.1. The sum divisor cordial labeling of Swastik graph $S w_{2}$ is shown in Figure 1.

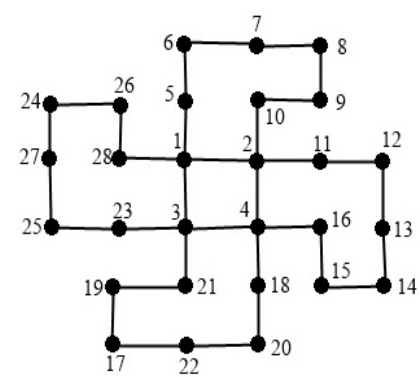

Figure 1: Sum divisor cordial labeling of Swastik graph $S w_{2}$

Theorem 2.2.The path union of finite copies of the Swastik graph $S w_{n}$ is a sum divisor cordial graph. 
Some New Results on Sum Divisor Cordial Graphs

Proof: Let $G=P\left(k \cdot S w_{n}\right)$ be the path union of $k$ copies for the Swastik graph $S w_{n}$, where $k \geq 2$ is a positive integer. We denote the $r^{\text {th }}$ copy of $S w_{n}$ by $S w_{n}^{r},(1 \leq r \leq k)$. Let $V\left(S w_{n}^{r}\right)=\left\{v_{i, j}^{r}: 1 \leq i \leq 4 ; 1 \leq j \leq 4 n\right\}$, where $1 \leq r \leq k$. Note that each copy of $S w_{n}$ has $p=16 n-4$ vertices and $q=16 n$ edges. Join the vertices $v_{4,2 n+1}^{r}$ with $v_{4,2 n+1}^{r+1}$ for $r=1,2, \ldots, k-1$ by an edge to form the path union of $k$ copies of the Swastik graph. Finally, we observe that $|V(G)|=k(16 n-4)$ and $|E(G)|=(k-1)+k .16 n$.

We define the vertex labeling $f: V(G) \rightarrow\{1,2, \ldots,|V(G)|\}$ as follows.

$$
\begin{aligned}
& f\left(v_{1,1}^{r}\right)=(r-1) p+1=f\left(v_{4,4 n}^{r}\right) ; 1 \leq r \leq k, \\
& f\left(v_{2,1}^{r}\right)=(r-1) p+2=f\left(v_{1,4 n}^{r}\right) ; 1 \leq r \leq k, \\
& f\left(v_{3,1}^{r}\right)=(r-1) p+4=f\left(v_{2,4 n}^{r}\right) ; 1 \leq r \leq k, \\
& f\left(v_{4,1}^{r}\right)=(r-1) p+3=f\left(v_{3,4 n}^{r}\right) ; 1 \leq r \leq k, \\
& f\left(v_{1, j}^{r}\right)=(r-1) p+1+j+2 ; j=2,3, \ldots, 4 n-1, \\
& f\left(v_{2, j}^{r}\right)=(r-1) p+4 n+1+j ; j=2,3, \ldots, 4 n-1, \\
& f\left(v_{3, j}^{r}\right)=(r-1) p+2(4 n+j)-2 ; j=2,3, \ldots, 2 n, \\
& f\left(v_{3, j}^{r}\right)=(r-1) p+2(2 n+j)+1 ; j=2 n+1, \ldots, 4 n-1 .
\end{aligned}
$$

The vertices of $v_{4, j}^{r}(1 \leq r \leq k)$ are labeled from the following two cases:

Case 1: When $r \equiv 1(\bmod 4)$ (or) $r \equiv 2(\bmod 4)$.

$$
\begin{aligned}
& f\left(v_{4, j}^{r}\right)=(r-1) p+2(6 n+j)-4 ; j=2,3, \ldots, 2 n, \\
& f\left(v_{4, j}^{r}\right)=(r-1) p+2(4 n+j)-3 ; j=2 n+1, \ldots, 4 n-1 .
\end{aligned}
$$

Case 2: When $r \equiv 0(\bmod 4)$ (or) $r \equiv 3(\bmod 4)$.

$$
\begin{aligned}
& f\left(v_{4, j}^{r}\right)=(r-1) p+2(4 n+j)+3 ; j=2,3, \ldots, 2 n, \\
& f\left(v_{4, j}^{r}\right)=(r-1) p+2(3 n+j)+2 ; j=2 n+1, \ldots, 4 n-1 .
\end{aligned}
$$

From the above labeling pattern, we observe that $e_{f}(0)=e_{f}(1)$, when $n$ is odd and $e_{f}(1)=e_{f}(0)+1$, when $n$ is even. Hence $\left|e_{f}(0)-e_{f}(1)\right| \leq 1$.

Thus, $G$ is a sum divisor cordial graph.

Example 2.2. The sum divisor cordial labeling of path union of 3 copies of Swastik graph $S w_{2}$ is shown in Figure 2. 


\section{A.Sugumaran and K.Rajesh}

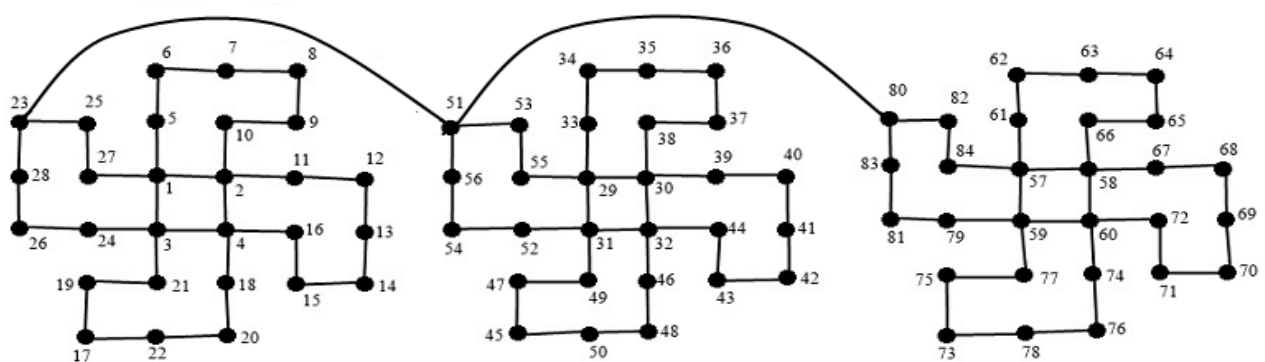

Figure 2: Sum divisor cordial labeling of path union of 3 copies of Swastik graph $S w_{2}$

Corollary 2.2.1. A cycle of $k$ copies of Swastik graph $C\left(k \cdot S w_{n}\right)$ is a sum divisor cordial graph, where $k$ is odd.

Proof: Let $G=C\left(k . S w_{n}\right)$ be a cycle of Swastik graph $S w_{n}$, where $k$ is odd positive integer. First we consider the path union of $k$ copies of $S w_{n}$. By Theorem 2.2, the path union of $k$ copies of $S w_{n}$ is a sum divisor cordial graph and $e_{f}(0)=e_{f}(1)$, since $k$ is odd. Now we obtain the cycle of $k$ copies of $S w_{n}$ from the path union of $k$ copies of $S w_{n}$ by joining an additional edge connecting the vertices $v_{4,2 n+1}^{1}$ and $v_{4,2 n+1}^{k}$. The label of this particular edge does not affect the condition $\left|e_{f}(0)-e_{f}(1)\right| \leq 1$. Hence $G$ is a sum divisor cordial graph.

Example 2.2.1. The sum divisor cordial labeling of $C\left(3 . S w_{2}\right)$ is shown in Figure 3 .

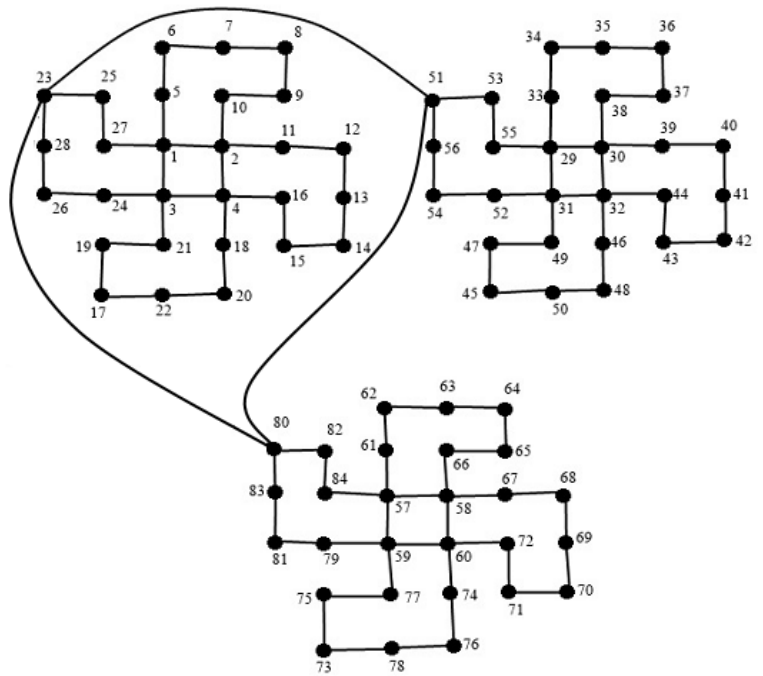

Figure 3: sum divisor cordial labeling of $C\left(3 . S w_{2}\right)$

Corollary 2.2.2. A cycle of $k$ copies of Swastik graph $C\left(k . S w_{n}\right)$ is a sum divisor cordial graph, where $k \equiv 0(\bmod 4)$. 
Some New Results on Sum Divisor Cordial Graphs

Proof: Let $k=4 m$, for some positive integer $m$. By Theorem 2.2, the path union of $k$ copies of $S w_{n}$ is a sum divisor cordial graph and $e_{f}(1)=e_{f}(0)+1$, since $k$ is even. We obtain the cycle of $k$ copies of $S w_{n}$ from the path union of $S w_{n}$ by joining an additional edge e (say) connecting the vertices $v_{4,2 n+1}^{1}$ and $v_{4,2 n+1}^{k}$. By definition of $\mathrm{f}$ (as defined in Theorem 2.2), $f\left(v_{4,2 n+1}^{1}\right)$ labeled as an odd integer, but $f\left(v_{4,2 n+1}^{k}\right)$ labeled as an even integer. Hence the additional edge e takes the value 0 . Thus in graph $G$, $e_{f}(0)=e_{f}(1)=\frac{1}{2}[(k-1)+k .16 n]$.

Hence, $C\left(k . S w_{n}\right)$ is a sum divisor cordial graph.

Remark 2.2. The graph $C\left(k . S w_{n}\right)$ is not a sum divisor cordial graph, if $k=2(\bmod 4)$. Note that the path union of $k$ copies of $S w_{n}$ is a sum divisor cordial graph, and $e_{f}(1)=e_{f}(0)+1$, since $k$ is even. To obtain a cycle of $k$ copies of $S w_{n}$, the new edge $\mathrm{e}$ (say) is included, which connects the vertices $v_{4,2 n+1}^{1}$ and $v_{4,2 n+1}^{k}$. By definition of $\mathrm{f}$ (as defined in Theorem 2.2), the vertex $v_{4,2 n+1}^{1}$ is labeled an odd integer and the vertex $v_{4,2 n+1}^{k}$ is labeled an odd integer. Hence the new edge e is labeled as 1 . In this case, the graph $C\left(k . S w_{n}\right)$ has satisfying $e_{f}(1)=e_{f}(0)+2$. Hence $C\left(k . S w_{n}\right)$ is not a sum divisor cordial graph.

Theorem 2.3. The Jelly fish $J(n, n)$ is a sum divisor cordial labeling for $n \geq 1$.

Proof: Let $G=J(n, n)$. Let $V_{1}=\left\{v_{i}: 1 \leq i \leq 4\right\}$ be the vertices of cycle $C_{4}$ and let $V_{2}=\left\{p_{i}, q_{i}: 1 \leq i \leq n\right\}$ be the pendant vertices, which are appending at $v_{2}$ and $v_{4}$ respectively. Then $G$ has $2 n+4$ vertices and $2 n+5$ edges.

We define the vertex labeling $f: V(G) \rightarrow\{1,2, \ldots,|V(G)|\}$ as follows.

$f\left(v_{1}\right)=1, f\left(v_{2}\right)=3, f\left(v_{3}\right)=2, f\left(v_{4}\right)=4$,

$f\left(p_{i}\right)=i+4 ; i=1,2, \ldots, n$,

$f\left(q_{i}\right)=n+i+4 ; i=1,2, \ldots, n$.

From the above labeling pattern, we have $\left|e_{f}(0)-e_{f}(1)\right| \leq 1$.

Hence, $G$ is a sum divisor cordial graph.

Example 2.3. The sum divisor cordial labeling of Jelly fish $J(4,4)$ is shown in Figure 4.

Theorem 2.4. Petersen graph is a sum divisor cordial labeling.

Proof: Let $u_{1}, u_{2}, u_{3}, u_{4}, u_{5}$ be the internal vertices and let $u_{6}, u_{7}, u_{8}, u_{9}, u_{10}$ be the external vertices of Petersen graph such that each $u_{i}$ is adjacent to $u_{i+5}, 1 \leq i \leq 5$. 


\section{A.Sugumaran and K.Rajesh}

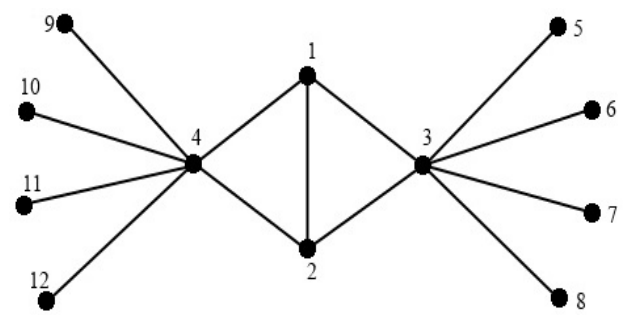

Figure 4: Sum divisor cordial labeling of Jelly fish $J(4,4)$

We define the vertex labeling $f: V(G) \rightarrow\{1,2, \ldots,|V(G)|\}$ as follows.

$f\left(u_{i}\right)=i ; 1 \leq i \leq 5, f\left(u_{6}\right)=10, f\left(u_{i}\right)=i-1 ; 7 \leq i \leq 10$.

From the above labeling pattern, we have $\left|e_{f}(0)-e_{f}(1)\right| \leq 1$.

Hence Petersen graph is a sum divisor cordial graph.

Example 2.4. The sum divisor cordial labeling of Petersen graph is shown in Figure 5.

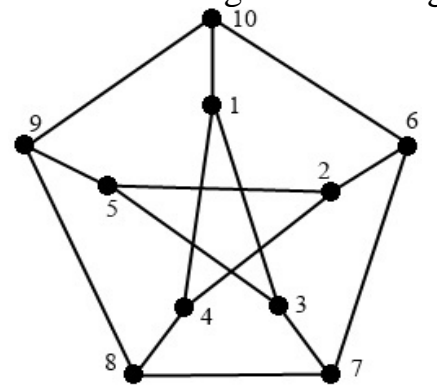

\section{Conclusion}

Figure 5: Sum divisor cordial labeling of Petersen graph

In this paper, we have proved that Swastik graph $S w_{n}$, path union of finite copies of Swastik graph $S w_{n}$, cycle of $k$ copies of Swastik graph $S w_{n}$ ( $k$ is odd), Jelly fish $J(n, n)$ and Petersen graph are sum divisor cordial graphs.

\section{REFERENCES}

1. R.Avudainayaki, B.Selvam and P.P.Ulaganathan, 3-Equitable and Total magic cordial Labeling for the extended duplicate graph of splitting graph of path, Annals of Pure and Applied Mathematics, 13 (2) (2017) 199-206.

2. J.A.Gallian, A dynamic survey of graph labeling, The Electronics Journal of Combinatorics, (2016).

3. L.Girija and A.Elumalai, Edge magic total labeling of the cycle $C_{n}$ with $P_{3}$ chords, Annals of Pure and Applied Mathematics, 8 (2) (2014) 175-181.

4. F.Harary, Graph Theory, Addison-Wesley, Reading, Massachusetts (1972).

5. C.Jayasekaran and J.Little Flower, Edge trimagic total labeling of mobius ladder, book and dragon graphs, Annals of Pure and Applied Mathematics, 13 (2) (2017) 151-163. 
Some New Results on Sum Divisor Cordial Graphs

6. V.J.Kaneria and H.M.Makadia, Graceful labeling for Swastik graph, International Journal of Mathematics and its Applications, 3 (2015) 25-29.

7. V.Lakshmi Alias Gomathi, A.Nellai Murugan and A.Nagarajan, Some results on elegant Graphs, International Journal of Mathematical Archive, 3 (2012) 1017-1028.

8. A.Lourdusamy and F.Patrick, Sum divisor cordial graphs, Proyecciones Journal of Mathematics, 35 (1) (2016) 115-132.

9. A.H.Rokad and G.V.Ghodasara, Fibonacci cordial labeling of some special graphs, Annals of Pure and Applied Mathematics, 11 (1) (2016) 133-144.

10. Ujwala Deshmukh and Vahida Y Shaikh, Mean cordial labeling of Tadpole and olive Tree, Annals of Pure and Applied Mathematics, 11 (2) (2016) 109-116.

11. S.K.Vaidya and N.H.Shah, Some star and Bistar related Divisor cordial graphs, Annals of Pure and Applied Mathematics, 3 (1) (2013) 67-77.

12. R.Varatharajan, S.Navaneethakrishnan and K.Nagarajan, Divisor cordial graph, International J. Math. Combin. 4 (2011) 15-25. 